\title{
Centrality Measures to Identify Traffic Congestion on Road Networks: A Case Study of Sri Lanka
}

\author{
I.M.L.N. Jayaweera ${ }^{1}$, K.K.K.R. Perera ${ }^{2}$, J. Munasinghe ${ }^{3}$ \\ 1, 2, 3 (Department of Mathematics, Faculty of Science, University of Kelaniya, Sri Lanka)
}

\begin{abstract}
This study presents a graph theoretical approach to identify the traffic congestion on a road network. Problem address on a city called Kiribathgoda situated in the western province of Sri Lanka. In the analysis of social networks, centrality measures played a vital role to identify the central nodes in a given network. We look at the applicability of centrality and betweenness measures in order to identify the most important locations which directly affect to the traffic congestion in road networks in Sri Lanka. Using the graph theoretical approach a traffic network for a selected area was constructed and several centrality measures were calculated. According to our simulation results, it was noted that the practically identified locations could be identified from the simulations carried out using the centrality measures.
\end{abstract}

Keywords- Centrality Measures, Betweenness Measures, Road Network, Graph, Traffic

\section{INTRODUCTION}

Traffic congestion has become a major problem that affects many human activities on a daily basis, resulting in both serious of transportation delays and also environmental damages. Therefore identifying the junctions of roads which directly involve in traffic congestion would be helpful to design the road networks. Since massive financial and man-hour loss due to traffic congestion, it becomes a major issue for all of us to analyze the traffic networks. In order to control traffic congestion, it is essential to understand the development of traffic flows. Many relations in real world problems can be represented by graph networks, in which the data are represented by nodes and the relationship between nodes are represented by links. Web graphs, internet graphs, communication networks, biological networks such as food web are some of the examples of the existing networks [1]. One of the main goal of network analysis is to determine the most important nodes in a given network. Several measures of importance have been studied in the literature to quantify how much a node is important. The objective of this problem is to identify the most important locations in the network and design the routes in order to control the traffic congestion. Most authors have been analyzed road networks from the viewpoints of shortest path, cost minimization etc. In 1995, Yang. H. et.al, introduced a model for determining traffic assignment and optimizing signal timings in road networks [2]. In [3], the relationship between centrality measures and the density of traffic for some simple particle hopping models on networks was identified with emerging scale-free degree distributions. Network representation was used to analyze the patterns in a street [4]. An efficient algorithm to find the shortest route between two nodes of a large scale, time-dependent graph were developed on road network [5]. Cut-set of a graph were used to find the optimal control of the traffic system [6]. However, centrality measures were considered by very few authors to analyze the traffic flows. We used centrality measures to analyze one way road network constructed for small area in Sri Lanka [7]. In this study, we use the centrality measures to analyze the congestion on the two way road networks in Sri Lanka based on directed graphs. Advantages of centrality measures are that it can provide a useful measurement to identify the importance of nodes, intersections and also relation between road segments. In order to test the validity, we construct a road network using most possible number of routes which covered an area between Thorana junction and Kiribathgoda junction. Constructing two networks as undirected graphs based on one way routes from Thorana junction to Kiribathgoda junction and Kiribathgoda junction to Thorana junction, we construct a large directed network which represent the actual road network near Kiribathgoda city. This study is based on four centrality measures named Degree, Closeness, Betweenness and Eigenvector, which are mainly used to analyze the social networks such as food web, internet graphs, and biological networks etc. These measures are used to compute the centrality of road networks. By considering centrality measures, we could identify the most important nodes and the road segments in our road network. It could also be verified that our computed centrality measures are useful to identify the traffic congestion in our road network. Correlation analysis to compare the shortest path betweenness and random walk betweenness were also carried out. Clustering analysis of the networks are useful to identify the distribution of the road networks and measure the density of the traffic. The rest of the paper is organized as follows. Definitions and notations of the centrality measures are given in Section 2. The methodology is outlined in Section 3 and the implementation results are discussed in Section 4. Finally, the conclusion is stated in Section 5. 
2.1 Centrality Measures

II.

DEFINITIONS

In this section, the definitions of centrally measures which used throughout the paper is summarized.

Definition 1 Let $v$ be a node and $\operatorname{deg}(v)$ be the degree of a node $v$. Then the degree centrality $C_{D}(v)$ is defined as $[2,8]$

$$
C_{D}(v)=\frac{\operatorname{deg}(v)}{n-1}
$$

where $n$ is the number of nodes in a network.

Definition 2 Closeness centrality $C_{c}(v)$ is defined as the mean geodesic (shortest path) distance between a vertex and all other vertices reachable from it $[1,9]$.

The closeness centrality $C_{c}(v)$ for vertex $v$ is defined as

$$
C_{c}(v)=\frac{\sum_{u \in V \backslash v} d(u, v)}{n-1},
$$

where $n$ is the number of nodes in the network and $d(u, v)$ is the shortest distance from $u$ to $v$.

Definition 3 Shortest Path Betweenness is defined as the number of shortest paths between pair of vertices passing through a vertex $v$ [10]. Then the formal definition of the betweenness of a vertex $v$ can be expressed as

$$
c_{B}(v)=\sum_{s \neq v \neq t \in V} \frac{d(s, t \mid v)}{d(s, t)},
$$

where $d(s, t \mid v)$ is the number of shortest paths between $s$ and $t$ passing through vertex $v$ and $d(s, t)$ is the total number of shortest paths between $s$ and $t$.

Similarly, edge betweenness of an edge can be defined as

$$
C_{B}(e)=\sum_{s_{s} t \in V} \frac{d(s, t \mid e)}{d(s, t)},
$$

where $d(s, t \mid e)$ is the number of shortest paths between $s$ and $t$ passing through edge $e$ and $d(s, t)$ is the total number of shortest paths between $s$ and $t[1]$.

Definition 4 Random Walk betweenness of a vertex $i$ is equal to the number of times that a random walk starting at $s$ and ending at $t$ passes through $i$ along the way, averaged over all $s$ and $t$. It does not only count the shortest paths passing through the given node, but all the other possible paths crossing it [10]. Then the average flow over all source-target pairs is given by,

$$
b(i)=\frac{\sum_{s<t} I_{i}^{(s t)}}{\frac{1}{2} n(n-1)},
$$

where $I_{i}^{(s t)}=\frac{1}{2} \sum_{j} A_{i j}\left|T_{i s}-T_{i t}-T_{j s}+T_{j t}\right|$, for $i \neq s, t$ and $I_{s}^{(s t)}=1, I_{t}^{(s t)}=1$. Here, $A$ denotes the adjacency matrix and $T$ is a generated matrix by removing last row and column from the Laplacian matrix and then back substitution of zero rows and columns into inverse Laplacian matrix.

Definition 5 Eigenvector centrality is a measurement of the centrality of a node in a network based on the weighted sum of centralities of its neighbors. It could be helpful to identify the nodes in the network that are connected to many other well-connected nodes [11].

$$
x_{i}=\frac{1}{\lambda_{1}} \sum_{j=1}^{N} A_{i j} x_{j}
$$

Definition 6 Network Criticality is the average (normalized) network criticality quantifies the average criticality of any two pairs of nodes in a network $[12,13]$ and given by $\tau=2 n \operatorname{Tr}\left(L^{+}\right)$, where $L^{+}$is Moore-Penrose inverse of Laplacian matrix. 
If $\tau$ of a specific network topology is less than another network topology, then it is said to be more robust network.

Definition 7 Clustering Coefficient is a local property, which describes the network structure of nodes and measures the number of triangles in a graph.

Advantages of those measures are summarized in Table 1[10, 14].

Table 1: Measures on Road Network

\begin{tabular}{|l|l|}
\hline \multicolumn{1}{|c|}{ Measure } & \multicolumn{1}{c|}{ Purpose } \\
\hline $\begin{array}{l}\text { Degree Centrality } \\
\text { DC) }\end{array}$ & $\begin{array}{l}\text { To measure the extent that a road segment is connected to all } \\
\text { segment in a network. }\end{array}$ \\
\hline $\begin{array}{l}\text { Closeness } \\
\text { Centrality (CC) }\end{array}$ & $\begin{array}{l}\text { To measure the extent that a road segment is near to all segments } \\
\text { in the road network along the shortest paths. It also captures the } \\
\text { notion of accessibility of a place at the city scale. }\end{array}$ \\
\hline $\begin{array}{l}\text { Shortest Path } \\
\text { Betweenness } \\
\text { (SPB) }\end{array}$ & $\begin{array}{l}\text { To capture the idea that a road segment is central if it lies on the } \\
\text { shortest paths that links all segments with each other. }\end{array}$ \\
\hline $\begin{array}{l}\text { Edge Betweenness } \\
\text { (EB) }\end{array}$ & $\begin{array}{l}\text { To capture the edges in the network that are crucial for } \\
\text { information flows. }\end{array}$ \\
\hline $\begin{array}{l}\text { Random Walk } \\
\text { Betweenness } \\
\text { (RWB) }\end{array}$ & $\begin{array}{l}\text { To find the number of times a vertex appears on a random walks } \\
\text { between all pairs in the network. }\end{array}$ \\
\hline $\begin{array}{l}\text { Eigenvector } \\
\text { Centrality (EC) }\end{array}$ & To measure the influence of nodes in road network. \\
\hline
\end{tabular}

\section{METHODOLOGY}

In this study, we selected a small area around Kiribathgoda city situated in the western province of Sri Lanka, which contains a heavy traffic jam during school and office hours. Many paths from Thorana Junction to Kiribathgoda as well as from Kiribathgoda to Thorana junction in Colombo-Kandy main road and other sub paths were considered to construct a road network. Our previous study was focused on undirected graph network, which consists of one way road networks [7]. In the present study road network was constructed as a weighted directed graph, where each node represents an intersection, junction, or a special place and each edge represent a road segment between those intersections. Weights of edges are taken as the distances between nodes (Data are collected from Google Map). Directed network was constructed by merging two undirected graphs, where each graph represent the one way road. Due to the complexity of the networks, 118, 54 and 168 nodes were chosen to construct these three networks. All centrality measures (Degree, Closeness, Betweenness and Eigenvector) and network criticality for all nodes in those road networks were computed. Besides that clustering coefficients were also calculated. All simulations are carried out using Mathematica and MATLAB.

\section{RESULTS AND DISCUSSIONS}

In this section results from simulations are presented and the behavior of the road networks are discussed. Several types of centrality measures which introduced in section 3 for network 1 and network 2 are given in Figure 1 and Figure 2 respectively. According to the Figure 1(a) and Figure 2(a), nodes around the Kiribathgoda Hospital, Lord Buddha statue junction and Tyre junction represent a traffic congestion. According to the Figure 1(b) and 2(b), the node 71 (Junction of New Hunupitiya road) and nodes from Commercial bank to Dasa bus stop have the highest closeness centrality, since those are the most accessible nodes from the source nodes on two networks respectively. Looking at Figure 1(c) and Figure 2(c), we noted that nodes 73, 71, 72 (nodes are from New Hunupitiya junction to Kiribathgoda junction) have the highest betweenness centrality. Node 15 (in front of New Hunupitiya road) of the main road of network 2 has the highest centrality and nodes 14, 13 (nodes of Kiribathgoda junction and Makola junction) have the high betweenness centrality. This concludes that the road segments from New Hunupitiya road junction to Kiribathgoda junction is an important part in our network and also nodes belongs to this segment, are crucial to maintain node connections. Figure 1(e) and Figure 2(e) show that the nodes 100,102, 97 (nodes around the hospital) and nodes around Dalugama area have high eigenvector centrality. This concludes that the intersections around the hospital and Dalugama junction are well connected. 
Table 2: Correlation coefficients for the centrality positions of the Road Network 1

\begin{tabular}{|c|c|c|c|c|c|}
\hline & DC & CC & SPB & RWB & EC \\
\hline DC & 1.0000 & 0.1201 & 0.0100 & 0.0825 & 0.1845 \\
\hline CC & 0.1201 & 1.0000 & 0.1792 & 0.2783 & 0.0086 \\
\hline SPB & 0.0100 & 0.1792 & 1.0000 & 0.7891 & 0.0416 \\
\hline RWB & 0.0825 & 0.2783 & 0.7891 & 1.0000 & 0.0416 \\
\hline EC & 0.1845 & 0.0086 & 0.0416 & 0.0416 & 1.0000 \\
\hline
\end{tabular}

Table 3: Correlation coefficients for the centrality positions of the Road Network 2

\begin{tabular}{|c|c|c|c|c|c|}
\hline & DC & CC & SPB & RWB & EC \\
\hline DC & 1.0000 & 0.0571 & 0.1797 & 0.2967 & 0.2528 \\
\hline CC & 0.0571 & 1.0000 & 0.2955 & 0.3562 & 0.5870 \\
\hline SPB & 0.1797 & 0.2955 & 1.0000 & 0.8447 & 0.2521 \\
\hline RWB & 0.2967 & 0.3562 & 0.8447 & 1.0000 & 0.3752 \\
\hline EC & 0.2528 & 0.5870 & 0.2521 & 0.3752 & 1.0000 \\
\hline
\end{tabular}

As indicated in Figure 3, it was observed that New Hunupitiya road junction is the most important node in the network and the road segment from New Hunupitiya road junction to Makola junction is an important part of our network.

\subsection{Correlation Coefficients}

Correlation coefficients for the centrality measures of the road networks are given in the Table 2 and Table 3.

According to Table 2 and Table 3, it is clear that the shortest path betweenness and random walk betweenness is highly correlated $(79 \% \& 84 \%)$, while other measures are weakly correlated in networks $1 \& 2$ respectively. Thus, in general, vertices with higher shortest path betweenness tend to have high random walk betweenness.

4.2 Statistical Analysis

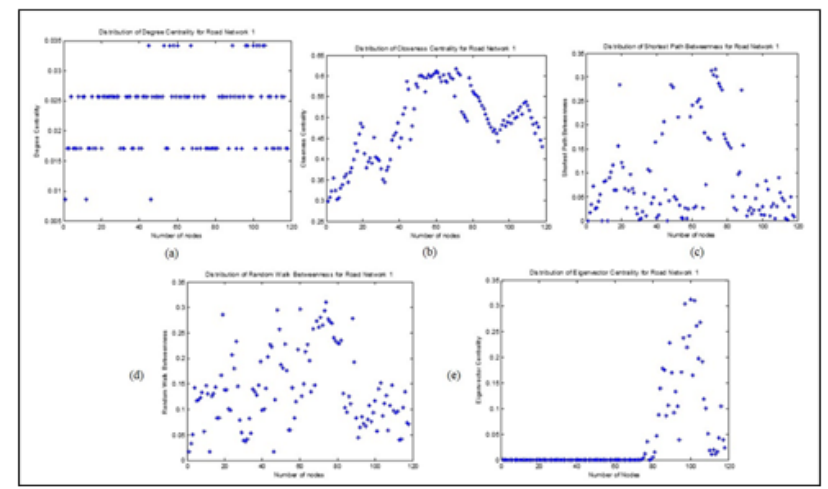

Figure 1: Centrality and Betweenness Measures for network 1 


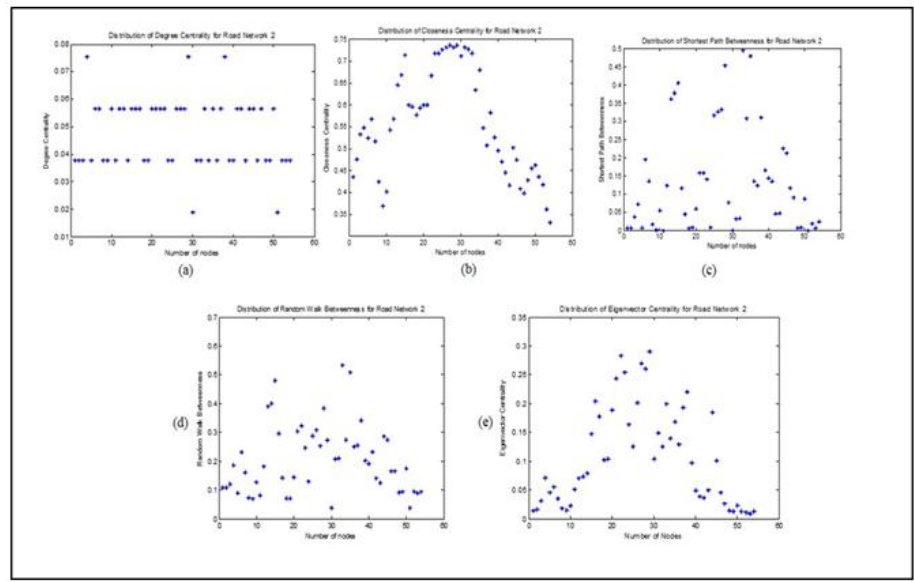

Figure 2: Centrality and Betweenness Measures for network 2

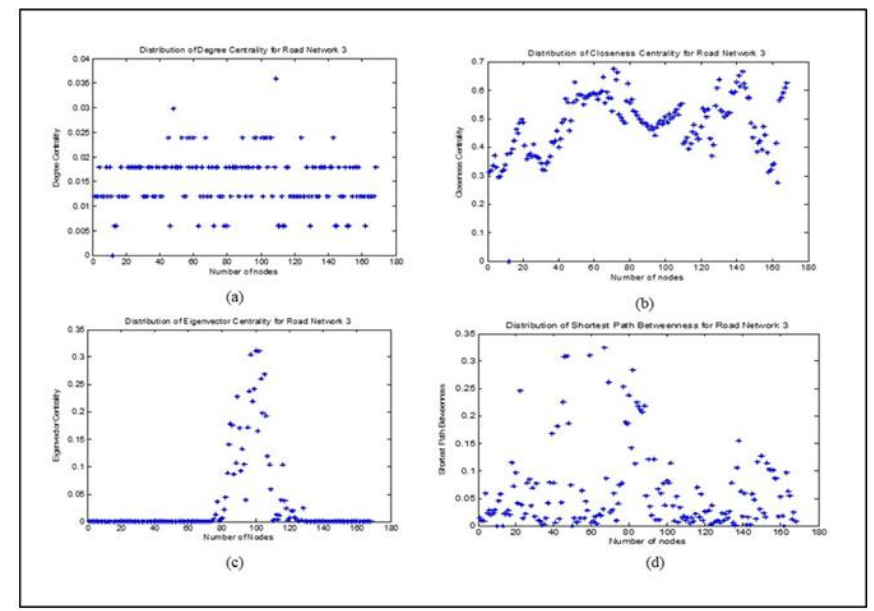

Figure 3: Centrality and Betweenness Measures for network 3

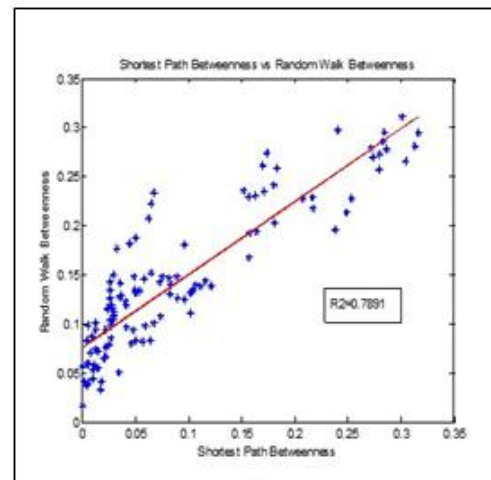

(a)

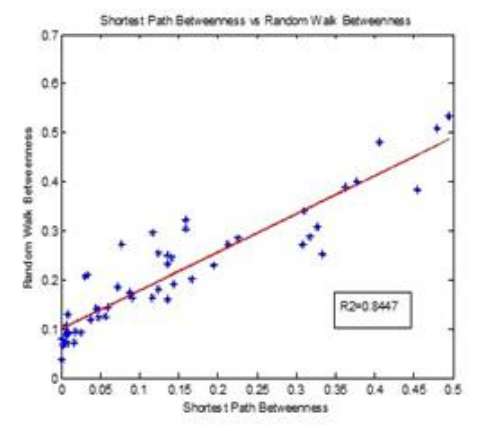

(b)

Figure 4: Correlation between shortest path betweenness against random walk betweenness for road network 1 \& 2 


\subsection{Clustering Coefficient}

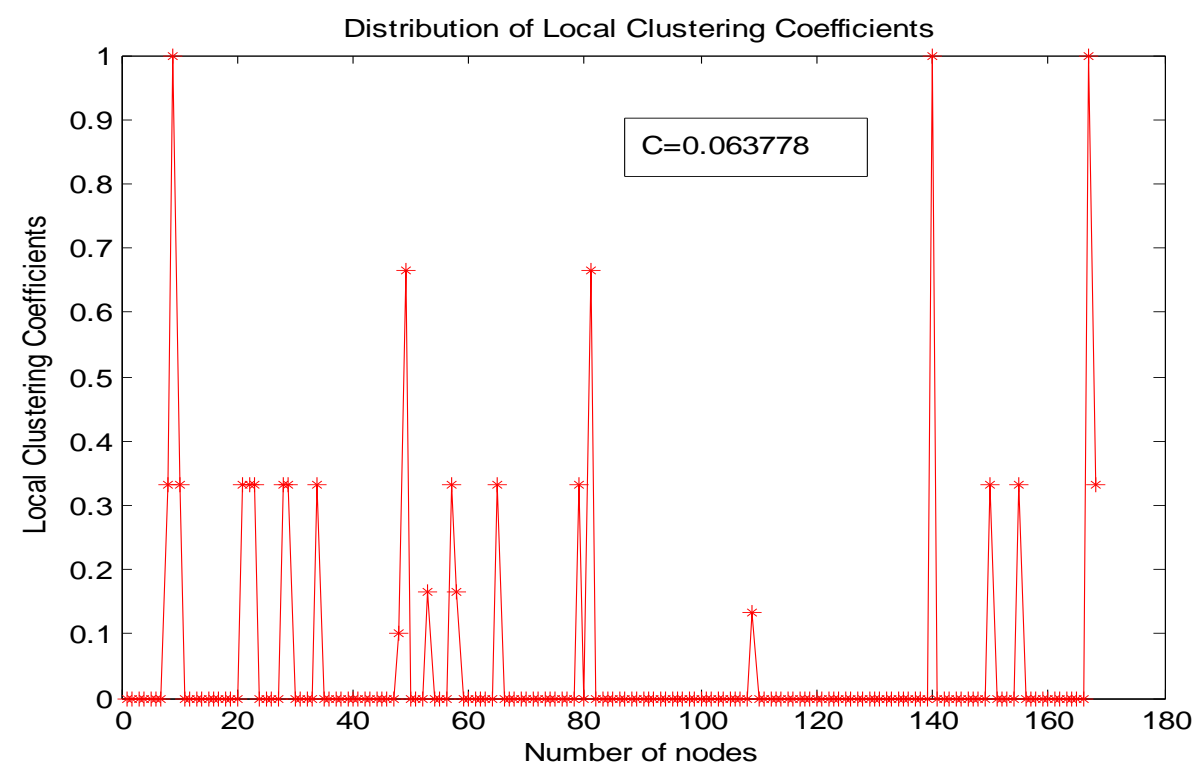

Figure 5: Local Clustering Coefficients for road network 3

Table 4: Network criticality of Road Networks

\begin{tabular}{|c|c|c|c|}
\hline & Number of nodes & Number of edges & Network Criticality \\
\hline Network 1 & 118 & 160 & 41.79 \\
\hline Network 2 & 54 & 68 & 28.86 \\
\hline Network 3 & 168 & 425 & 34.19 \\
\hline
\end{tabular}

The overall clustering coefficient of the first, second and third road networks are only 0.037681 , 0.044872 and 0.063778 (approximately equal to zero), indicating a poor aggregation, which is consistent with the cluster and long-strip distribution of the road network and has low density. The overall clustering coefficient of the first, second and third road networks are only $0.037681,0.044872$ and 0.063778 (approximately equal to zero), indicating a poor aggregation, which is consistent with the cluster and long-strip distribution of the road network and has low density.

\subsection{Network Criticality}

Network Criticality of the three networks are measured and listed in the Table 4. According to the Table 4, it can be noted that the network 2 is more robust than the network 1 .

\section{CONCLUSIONS}

In this paper, we addressed the problem of identifying the important parts of a traffic network, which may be helpful to reduce the traffic congestion. By selecting a small area of a road network (from Thorana junction to Kiribathgoda) in Sri Lanka, we constructed a road traffic network as a directed weighted graph. Then the centrality and betweenness measures were calculated. According to the results we obtained, we concluded that New Hunupitiya road junction is the most valuable node in our road network. Besides that the road segment from University of Kelaniya to Kiribathgoda junction is an important road segment in our network. We noticed that New Hunupitiya road Junction is the most valuable node in this network and there should be a traffic light to control the traffic on road network. Nodes which we identified from the simulation results are very important to reduce the traffic congestion accurately.

ACKNOWLEDGEMENT

Laboratory facilities provided by the Department of Mathematics of the University Of Kelaniya is acknowledged. 


\section{REFERENCES}

[1]. M. Girvan and M.E. Newman, Community structure in social and biological networks: In Proceedings of the National Academy of Sciences. 2002.99(12): 7821-7826.

[2]. H. Yang and Y. Sam, Traffic assignment and signal control in saturated road networks. Transportation Research Part A. Policy and Practice. 1995. 29(2):125-139.

[3]. P. Holme, Congestion and centrality in traffic flow on complex networks. Advanced in Complex Systems. 2003. 6(2): 163-176.

[4]. P. Masucci, D. Smith, A. Crooks and M. Batty, Random Planar graphs and London Street network (2004).

[5]. G. Nannicini, P. Baptise, D. Krob and L. Liberti, Fast Computation of point-to-point paths on time dependent road networks. Combinatorial Optimization and Applications. 2008. 225-234.

[6]. N. Baruah and A.K. Baruah, On a Traffic Control Problem Using Cut-Set of Graph. Int. J. Advanced Networking and Applications. 2012. 3(4): 1240- 1244.

[7]. I.M.L.N. Jayaweera and K.K.K.R. Perera, A Graph theoretic approach to control the traffic congestion on road networks, In Proceedings of the Research Symposium of Uva Wellassa University, Sri Lanka, 2015. $1-3$.

[8]. L.C. Freeman, Centrality in social networks conceptual clarification. Social Networks. 1978/1979. 1(3): 215-239.

[9]. E.W. Dijkstra, A note on two problems in connection with graphs. Numerische Mathematik. 1959. 1(1): 269-271.

[10]. M.E.J. Newman, A measure of betweenness centrality based on random walks. Social networks. 2005. 27.1: 39-54.

[11]. M.W. Newman, The Laplacian Spectrum of Graphs. PhD thesis, Department of Mathematics, University of Manitoba. 2000.

[12]. S.B. Dennis, Matrix Mathematics. Princeton University Press. 2005.

[13]. A. Tizghadam and A. Leon-Garcia, A Graph Theoretic Approach to Traffic Engineering and Network Control Problem, In Teletraffic Congress, ITC 21 2009, $21^{\text {st }}$ Internationals. 2009. 1-8.

[14]. A.B. Jayasinghe and N. Pathiranage, 'Centrality Measures' as a tool to identify the transit demand at railway stations: a case of railway network, Sri Lanka. SAIIM Research Symposium on Engineering Advancements, 2013. 16-19. 